\title{
Octopamine Signaling Via Oamb is Essential for A Well-Orchestrated Climbing Performance of Adult Drosophila Melanogaster
}

Samar Ezzat El-Kholy ( $\sim$ samar_elkholy@science.tanta.edu.eg )

Tanta University

Basma Afifi

Tanta University

Iman El-Husseiny

Tanta University

Amal Seif

Tanta University

\section{Research Article}

Keywords: Octopamine, oamb, Drosophila, climbing performance, skeletal muscles.

Posted Date: December 30th, 2021

DOI: https://doi.org/10.21203/rs.3.rs-1194811/v1

License: (c) (i) This work is licensed under a Creative Commons Attribution 4.0 International License. Read Full License 


\section{Abstract}

The biogenic amine octopamine (OA) orchestrates many behavioural processes in insects. OA mediates its function by binding to $O A$ receptors belonging to the $G$ protein-coupled receptors superfamily. Despite the potential relevance of OA for controlling locomotion, our knowledge about the role of each octopaminergic receptor still limited. In this study, RNA interference (RNAi) was used to knockdown each OA receptor type in almost all Drosophila melanogaster tissues using a tubP-GAL4 driver to investigate the loss of which receptor affects the climbing ability of adult flies. The results demonstrated that oambdeficient flies had impaired climbing ability more than those deficient in other receptors receptive for OA. Targeted RNAi-mediated kockdown of oamb in the nervous system or muscular system decreased the climbing ability, indicating that within Drosophila legs, OA through oamb orchestrated the nervous system control and muscular tissue responses. Oamb-deficient adult males showed morphometric changes in the length and width of leg parts. Transmission electron microscopy revealed that the leg muscles oambdeficient flies have severe ultrastructural changes compared to those of control flies. The severe impairment in the climbing performance of oamb-deficient flies correlates well with the completely distorted leg muscle ultrastructure in these flies. Taken together, we could conclude that OA via oamb plays an important role in the locomotor activity of Drosophila.

\section{Introduction}

Locomotor activity is an integrative characteristic of the functional state of the nervous system as it is implicated directly or indirectly in most types of insect behaviours such as foraging and mating. Distinct brain structures like the mushroom bodies or the central complex have been shown to be required for the control of locomotor activity (Martin et al. 1998; Strauss and Heisenberg 1993). In adult Drosophila, locomotor activity can be measured by different tools. Negative geotaxis performance is an innate escape behaviour in which adult flies ascend food vials as a response to tapping the vials. It could be used as a tool to evaluate the locomotor activity of Drosophila. This behaviour requires nervous system signaling commands via different biogenic amines.

Octopamine (OA) is one of these biogenic amines that acts as a neurohormone, neurotransmitter and neuromodulator in invertebrates. This catecholamine was first discovered in the salivary glands of Octopus vulgaris (Erspamer and Boretti 1951). OA is synthesised from the amino acid tyrosine. Tyrosine is converted by decarboxylation to tyramine by the enzyme tyrosine decarboxylase, and then tyramine is converted by hydroxylation to $\mathrm{OA}$ with the help of tyramine beta-hydroxylase. OA orchestrates many diverse physiological and behavioural processes including aggression, fight-flight response, circadian rhythm and locomotion (Roeder 1999). OA is essential for modulating the function of skeletal and visceral muscles (Orchard and Lange 1987). OA in invertebrates has the same function of noradrenaline in vertebrates. $O A$ exerts these functions by binding to and activating $O A$ receptors belonging to the $G$ protein-coupled receptor superfamily. Drosophila possesses four OA receptors: Oa2 (also referred as Octß1R : CG6919), oamb (CG3856), Octß2R (CG33976, CG6989) and Octß3R (CG42244, CG7078) (Maqueira et al. 2005). OA stimulates glycogenolysis, modifies muscle contraction, supports long-term 
flight and regulates "arousal" in the central nervous system (Brembs et al. 2007). Despite the potential relevance of $\mathrm{OA}$ for controlling locomotion, our knowledge about the role of each octopaminergic receptor still limited.

The GAL4/UAS system enables scientists to study either gain-of-function or loss-of-function of the gene (s) of interest (Brand and Perrimon 1993), facilitating studying gene expression in Drosophila (Fischer et al. 1988). The GAL4/UAS system has been used in RNA interference (RNAi) technology. This technology enables the knocking down or knocking out a specific gene of interest.

The main goal of this work was to reveal which one of the Drosophila OA receptors is important for adult locomotion. We achieved this by knocking down each OA receptor everywhere within the insect body using a universal driver and performing the negative geotaxis assay with the corresponding adults. Next, we explored whether the effect of OA originates from its effect on the nervous system or muscular system, and specific GAL4 drivers were crossed to OA receptor (s) followed by evaluation of the climbing ability performance of F1 male adults. Finally, we examined the morphometric and skeletal muscle ultrastructure changes in the legs of adult flies that displayed the knocking down of OA receptors. The findings of the current study provide evidence that $\mathrm{OA}$ via oamb orchestrates nervous system commands with muscular system response, consequently controlling balanced locomotion. Moreover, the current study is the first that link the leg muscles development with neurohormonal control. Information regarding biogenic amine receptors is very important for comparative, evolutionary physiology and biochemistry as well as serving as possible specific targets for insecticides.

\section{Results}

Crossings of three GAL4 lines with each OA receptor were used to reliably dissect the most important receptor in negative geotaxis performance. First, RNAi targeted against the corresponding receptor genes was crossed with the tubulin promoter GAL4-driver line tubP-GAL4 that drives expression in most organs of the fly. Data obtained revealed that despite all receptors receptive for OA have significant adverse effect on climbing performance, oamb -deficient flies showed the most severe impairment, which was highly significantly different $(P=0.005)$ from that of control flies (Fig. 1$)$. The control male flies showed the native characteristic strait-line walking behaviour to climb the cylinder. By contrast, oamb-deficient male flies showed impairment in moving up against gravity. Some flies attempted several times to climb up the cylinder for a short distance, but they failed to maintain their equilibrium and fell into the bottom of the cylinder. Furthermore, few flies showed complete defective locomotion behaviour. They remained on the cylinder bottom walking very slow throughout the experiment. Therefore, we focused on whether the underlying mechanism of octopamine signalling via oamb in climbing performance originated from the nervous system or muscular system. To achieve this, the oamb RNAi line was crossed to elav-GAL4 and Mhc-GAL 4 lines, respectively. The results showed that targeting the RNAi effect of oamb either to the nervous system or muscular system significantly $(P \otimes 0.001)$ decreased the climbing ability of adult males compared with control flies (Fig. 2A,B). Because oamb-deficient flies had the weakest performance in climbing ability, we focused on the morphometric changes in oamb-deficient adult leg parts. 
The length and width of each leg segment were measured. The results indicated that the lengths of all parts of forelegs $(P=0.001,0.006$ for coxa and trochanter ; $P=0.000$ for femur, tibia and tarsi)

and hind legs ( $P=0.000$ for coxa, trochanter, femur; $P=0.046,0.047$ for tibia and tarsi respectively). oamb-deficient flies were significantly different from those of control flies. In addition, the width of the trochanter and femur of the forelegs $(P=0.000,0.002$ for trochanter and femur respectively) and the coxa, trochanter and femur of the hind legs were significantly $(P=0.000,0.002,0.032$, respectively) different from those control flies (Fig. 3).

In this study, ultrastructure examination of the metathoracic leg of control flies revealed that the tubular oblique muscles consist of spindle-shaped myofibrils, arranged in parallel and had the known striated pattern of successive sarcomeres (Fig. 4A \& B). All muscle fibres were ensheathed in a sarcolemma. The sarcolemma invaginates into fibres at regular distances. Each sarcomere extends between adjacent Zdiscs of the myofibril. The $Z$ line generally looks like a series of dense bodies and disconnected through the sarcomere. Their roundish mitochondria are present between the myofilaments and contain an electron-dense matrix. In oamb-deficient flies, the muscles displayed disorganisation of all components. The sarcomeres and the entire myofibrils showed loss of striations and structure (Fig. 4C \& D). The sarcoplasmic reticulum around each myofibril was noticeably disintegrated such that division of the muscle fibres into myofibrils was indistinct. The myofibrils broke down such that there were spaces in the muscle fibre. The $Z$ line split into smaller fragments that were dispersed among the sarcomeres. Although mitochondria were distributed along damaged myofibrils, some were enlarged with an irregular contour, some were small with a rounded contour and others were absent from certain areas of the muscle fibre. Their cristae were electron lucent.

\section{Discussion}

OA is a catecholamine that acts as a neuromodulator in invertebrates (David and Coulon 1985; Roeder 1999, 2002, 2005). The role of neuromodulators was explained by "orchestration hypothesis," which assumed that neuromodulator release into specific neuropils configures neural commands to produce a coordinated network activity (Sombati and Hoyle 1984). OA mediates its function by binding to $G$ proteincoupled receptors, which included cyclic AMP production or $\mathrm{Ca}^{2+}$ release. OA regulates many physiological processes including aggression, fight-flight response, circadian retime and locomotion (Riemensperger et al, 2005; Schwaerzel et al, 2003; Unoki et al, 2005; Zhou et al, 2008).

Each leg segment of the multi-jointed legs of adult insects contains a stereotyped arrangement of muscles. Contractions of these muscles through motor neurons control the coordination of locomotion. OA plays an important role in this process. In locusts, $O A$ is delivered in time for enhancement of leg muscle contraction (Duch et al. 1999). In this study, we focused on the role played by octopaminergic receptors in balanced locomotion using RNAi of each receptor with three GAL4 drivers. Targeted RNAimediated knockdown using the tubulin-promoter GAL4 driver showed that oamb-deficient flies had the weakest performance in the negative geotaxis assay. The present study revealed significant impairment 
in negative geotaxis performance when oamb deficiency was directed to the muscular or nervous system. The walking speed of oamb-deficient flies was lower than that of control flies. Moreover, most of leg parts of oamb-deficient flies was smaller than those of control flies. These findings are interesting as they provide evidence that oamb acts in leg muscles as a neuromodulator/neurotransmitter that is important for normal leg muscle architecture and size and for the coordination of muscle contractions required for balanced locomotion. A possible explanation for these structural changes is based on the known physiological role of oamb mediated signalling that induces $\mathrm{Ca}^{2+}$ oscillations due to $\mathrm{Ca}^{2+}$ release from intracellular stores (Balfanz et al,2005). This reaction is controlled by kinase and phosphatase activities (Hoff et al, 2011). Furthermore, blocking phosphatase activity in oamb expressing cells completely abolished $\mathrm{Ca}^{2+}$ oscillations (LIT). In mammals, it has been shown that $\mathrm{Ca}^{2+}$-dependent pathways control muscle development (Damm and Egli, 2014). Lowering intracellular calcium levels inhibits the differentiation of skeletal myoblasts into mature myotubes (Porter et al, 2002). Moreover, it has been shown in mammals that adrenergic receptors signalling regulated myoblast differentiation (Saini et al, 2010; Church et al, 2014). This might explain, why the lack of oamb-mediated signalling events during development leads to the observed structural changes. The neuromodulatory role of OA in skeletal and visceral muscle contraction was reported before (Evans 1981). Oamb expression was reported in adult Drosophila leg muscles (El-Kholy et al. 2015). Oamb, the invertebrate counterpart of mammalian aadrenergic receptor, is also expressed in the oviduct muscles of female insects and regulates their contraction by elevating the cytosolic $\mathrm{Ca}^{+2}$ level (Lee et al. 2003) and in the tracheal system (El-Kholy et al. 2015). OA through oamb regulates other physiological processes such the induction of insulin release from insulinproducing cells causing changes in the amount of daily sleep and changes in fat storage, leading to lean adult flies (Crocker and Sehgal 2008; Crocker et al. 2010; Erion et al. 2012; Luo et al. 2014; Li et al. 2016).

To verify the role of oamb in the formation of normal leg muscle architecture, leg muscles of male Drosophila were analysed using transmission electron microscopy. The results revealed that in oambdeficient flies, the leg muscles displayed abnormal morphologies of sarcomeres, disorganised myofibrils and mitochondrial abnormalities. For these reasons, male Drosophila flies hypothesize to show severe defective locomotion behaviour. The sarcomeres and the entire myofibrils showed loss of striations and structure. The sarcoplasmic reticulum around each myofibril was noticeably disintegrated; consequently, division of the muscle fibres into myofibrils was indistinct. Moreover, the myofibrils broke down forming spaces in the muscle fibre. The thickness of myofibrils that could be observed in the leg muscles of oamb -deficient flies was significantly less than that the leg muscles of control flies.

Z-lines as anchoring structures for myofilaments dictate the final length of sarcomeres. Z-lines would be expected to participate in the organisation of myofilaments during the initial stages of myofibril assembly. The results of this study showed that Z-lines within the leg muscles of oamb-deficient flies were split into smaller fragments that were dispersed among the sarcomeres, causing loss of the normal muscle architecture and consequently impairment of the negative geotaxis performance of 
corresponding flies. Recently, Sujkowski et al. (2020) reported that oamb expression in Drosophila is required for adaptations in skeletal muscles in legs.

Mitochondria play an important role in the muscular system. In Drosophila, myofibers have a greater dependency on mitochondria and lipid oxidation and, thus, the numbers of healthy mitochondria can influence the capacity to maintain muscle mass and function. In skeletal muscles, mitochondria regulate energy haemostasis by producing ATPs required for muscle contraction through oxidative phosphorylation. In addition, mitochondria contribute to $\mathrm{Ca}^{+2}$ homeostasis (De Stefani et al. 2011; Eisner et al. 2014), redox signalling (Finkel 2011; Sena and Chandel 2012), release of pro-apoptotic factors (Frezza et al. 2006), synthesis of haeme molecule and regulation of nuclear gene expression (Picard et al. 2014; Chae et al. 2013). In this study, ultrastructural changes were detected in the shape of mitochondria within the leg muscles of oamb-deficient flies. The mitochondria were electron lucent, enlarged with an irregular contour or small with a rounded contour and others were absent from certain areas of the muscle fibre. These changes could be the reason behind the observed disorder of muscles.

Ultrastructure changes in mitochondria occur in muscle disorders (Kelley et al. 2002; Chen et al. 2010; Crane et al. 2010; Sao et al. 2010; Austin and St-Pierre 2012), particularly in muscular dystrophy, while in degenerating fibres, the number of mitochondria is reduced and they disappear from severely atrophied muscle fibres.

Taken together, the data presented in this study reveal that octopamine signalling via oamb plays an essential role in adult Drosophila movement ability as well as in normal leg muscle architecture formation and interorgan communication between the nervous system commands to motor neurons and muscular tissue responses. It became apparent that information regarding biogenic amine receptors is very important form many points of view including comparative, evolutionary physiology and biochemistry as well as serving as possible specific targets for insecticides.

\section{Materials And Methods}

\section{Drosophila strains and rearing}

All Drosophila lines were obtained from Bloomington Drosophila Stock Center (Bloomington Stock Centre, Indiana University, Bloomington, USA). These lines included RNAi effector lines generated by the Transgenic RNAi Project for the knockdown of Drosophila OA receptors and three GAL4 driver lines (tubPGAL4, elav-GAL4 andMhc-GAL4). UAS effector lines and GAL4 driver were mated to a $w 1118$ control line and each RNAi experiment was done with these 2 controls in parallel. At least two available constructs for the same receptor-coding gene have been tested to be sure the observed phenotype is not due to offtarget effects and the results presented in the manuscript were to the strongest construct which show high significant difference. All lines were reared on standard Drosophila medium (14-15 g agar, $18.5 \mathrm{~g}$ yeast, $61 \mathrm{~g}$ glucose, $30.5 \mathrm{~g}$ sucrose, $101 \mathrm{~g}$ corn meal/L) at $25^{\circ} \mathrm{C}$ except for $\mathrm{F} 1$ of the crossings was kept 
at $27-29{ }^{\circ} \mathrm{C}$ to enhance the RNA interference effect. All lines were kept at $50 \%-60 \%$ relative humidity with an 18/6-h light/dark cycle.

\section{Climbing assay (negative geotaxis)}

A negative geotaxis assay was performed using only male adult flies to avoid the effect of weight changes in adult female flies due to the physiological process including ovulation and oogenesis. Briefly, 10 males were placed into a $100 \mathrm{~mL}$ empty glass cylinder. The flies were tapped to the bottom of the cylinder and left to climb the cylinder. This procedure was repeated five times. The upward movement of the flies was recorded until the last climbing fly reached the top of the vial. The upward movement of flies was recorded with a digital video camera. The videos were cut into snapshots at the rate of 5 frames/second. These frames were analysed using ImageJ 1.46r software (National Institutes of Health, Bethesda, MD, USA) to trace the upward paths of the flies. The walking speed was calculated by dividing the length of the path (cm) by the time required in each path (s) (Rothenfluh and Heberlein 2000; Khuranaet al. 2010).

\section{Effect of OA receptor knockdown on climbing ability}

OA receptors were silenced using the GAL4/UAS system. Three GAL4 drivers were used. These were the universal driver tubulin promoter GAL4 (tubP-GAL4), which induces gene repression in almost all insect's body cells, nervous system-specific driver elav-GAL4 and muscular system-specific driver myosin heavy chain (Mhc-GAL4). Each one of these driver lines was independently crossed to the Oa2,oamb, Octß2R or Octß3R lines. The climbing ability of $10 \mathrm{~F} 1$ adult males was studied as described above. Adult F1 males of $w^{1118}$ crossed to - GAL4 and - UAS lines were used as controls.

\section{Morphometric analysis of adult legs}

Fore and hind legs were removed by forceps from adult flies and then transferred to glass slides using a fine brush for microscopic examination using an Olympus BX61 light microscope at magnification $2.5 \times 10$. Photographs were taken and the length and width of each leg segment (coxa, trochanter, femur, tibia and tarsi) were measured using ImageJ software.

\section{Transmission electron microscopy}

Ten hind legs were removed by forceps. Then, the legs were fixed in $5 \%$ glutaraldehyde in sodium cacodylate buffer ( $\mathrm{pH}$ 7.4) for 12-24 h. The specimens were washed and fixed overnight in 15\% buffered osmium tetroxide. Samples were incubated overnight in $0.5 \%$ aqueous uranyl acetate solution, then dehydrated, filtered and embedded in spur resin (Davidson 1981). Semi-thin (1 $\mu \mathrm{m})$ sections were stained with methylene blue Atur II and examined using an Olympus BX61 light microscope. Ultra-thin sections $(60 \mathrm{~nm})$ were cut using an ultramicrotome (Ultracut S, Leica), and stained with $2.5 \%$ uranyl acetate and lead citrate and were examined using JEOL, JEM 100-SX electron microscope. Sarcomere length (Z-Z 
distance), myofibril and sarcoplasmic reticulum thickness and mitochondrial area were measured using Image J software.

\section{Statistical analysis}

The normality of the obtained data was tested to check if data were parametric or non-parametric using Kolmogorov-Smirnov test. Statistical differences in means of different biological attributes were calculated by one-way ANOVA followed by student $t$-test. The level of significance was set at $P<0.05$ (Minitap 18.1 software).

\section{Declarations}

\section{ACKNOWLEDGMENTS}

The authors thank the staff members of the electron microscopy Unit, Mansoura University for their assistance in preparing the TEM samples.

\section{CONTRIBUTION TO THE FIELD}

Biogenic amines act as neurohormones, neuromodulators and neurotransmitters controlling many physiological processes in both vertebrates and invertebrates. Some commercially available pesticides act on biogenic amines such as Rotenone, neonicotinoids and formamidine . Biogenic amines-based pesticides have adverse effects on human, especially nervous system causing neurological diseases. For this reason, the biogenic amine octopamine and its related receptors represent good insecticide targets as being exclusive to invertebrates. Consequently, detailed information about the contribution of single receptor in vital physiological and behavioral processes may help to choose the most effective target for specific insecticides design tools. In this study, we focus on the contribution of octopaminergic receptors in locomotion, as a crucial behavior for survival. Results of tissue specific RNAi-based experiments revealed that oamb-deficient flies showed impaired negative geotaxis and leg muscles atrophy. In addition, this study revealed for the first time, the role of oamb in the development of normal leg muscles architecture. Previous studies stated that agonists and antagonists for octopamine receptors act as potential targets for insecticides (Evans and Maqueira, 2005), here our results specify OA receptor oamb for molecular docking for synthesis of specific insecticides due to the essential role of this receptor in locomotion and skeletal muscle development. In addition to this, the data provide new insights into what may be a potential future prospect for human muscle atrophy therapeutics by targeting the a-adrenergic receptors, the counterparts of invertebrate oamb.

\section{Data availability}

The datasets generated during and/or analysed during the current study are available from the corresponding author on reasonable request. 


\section{AUTHOR CONTRIBUTIONS}

SE-K suggested the study idea and designed the experiments, BA and SE-K performed experiments and evaluate the data, all authors wrote the manuscript.

\section{CONFLICT OF INTEREST}

The authors declare that the research was conducted in the absence of any commercial or financial relationships that could be construed as a potential conflict of interest.

\section{References}

1. Austin, S. and St-Pierre, J. (2012).PGC1a and mitochondrial metabolism-emerging concepts and relevance in ageing and neurodegenerative disorders. J. Cell Sci. 125, 49634971.doi:10.1242/jcs.113662.

2. Balfanz, S., Strunker, T., Frings,S. and Baumann, A. (2005). A family of octapamine receptors that specifically induce cyclic AMP production or $\mathrm{Ca} 2+$ release in Drosophila melanogaster. J. Neurochem. 2005, 93, 440-451. doi: 10.1111/j.1471-4159.2005.03034.x

3. Brand, A. H. and Perrimon, N. (1993).Targeted gene expression as a means of altering cell fates and generating dominant phenotypes.Development. 118(2), 401-415. doi:org/10.1242/dev.118.2.401

4. Brembs, B., Christiansen, F., Pfluger, H. J. and Duch, C. (2007). Flight initiation and maintenance deficits in flies with genetically altered biogenic amine levels. J. Neurosci. 27(41), 1112211131.doi:org/10.1523/JNEUROSCI.2704-07.2007

5. Chae, S., Ahn, B.Y., Byun, K., Cho, Y.M., Yu, M-H., Lee, B., et al. (2013).A systems approach for decoding mitochondrial retrograde signaling pathways. Sci. Signal. 26;6(264):rs4.doi: $10.1126 /$ scisignal.2003266.

6. Chen, H., Vermulst, M., Wang, Y. E., Chomyn, A., Prolla, T. A., McCaffery, J. M. and Chan, D. C. (2010). Mitochondrial fusion is required for mtDNA stability in skeletal muscle and tolerance of mtDNA mutations. Cell, 141, 280-289.doi: 10.1016/j.cell.2010.02.026

7. Church, JE, Trieu, J., Sheorey,R., Chee, AY.M., Naim,T., Baum,D.M., et al (2014). Functional $\beta$ Adrenoceptors Are Important for Early Muscle Regeneration in Mice through Effects on Myoblast Proliferation and Differentiation. PLOS.: e101379. doi:org/10.1371/journal.pone.0101379

8. Crane, J. D., Devries, M. C., Safdar, A., Hamadeh, M. J. and Tarnopolsky, M. A. (2010).The effect of aging on human skeletal muscle mitochondrial and intramyocellular lipid ultrastructure.J.Gerontol.A, Biol. Sci.Med.Sci. 65(A), 119-128.doi: 10.1093/gerona/glp179. Epub 2009 Dec 3.

9. Crocker, A. and Sehgal, A. (2008).Octopamine regulates sleep in Drosophila through protein kinase Adependent mechanisms. J. Neurosci. 28, 9377-9385.doi: 10.1523/JNEUROSCI.3072-08a.2008.

10. Crocker, A., Shahidullah, M., Levitan, I. B. and Sehgal, A. (2010). Identification of a neural circuit that underlies the effects of octopamine on sleep:wake behavior. Neuron. 65, 670-681. 
doi: 10.1016/j.neuron.2010.01.032

11. Damm, B. andEgli, M. (2014).Calcium's Role in Mechanotransduction during Muscle Development.Cell. Physiol. Biochem. 33,249-272.doi: 10.1159/000356667. Epub 2014 Jan 31.

12. David, J.C. and Coulon, J.F. (1985). Octopamine in invertebrates and vertebrates.Prog.Neurobiol. 24, 141-185.doi: 10.1016/0301-0082(85)90009-7.

13. De Stefani, D., Raffaello, A., Teardo, E., Szabo, I. and Rizzuto, R. (2011). A forty-kilodalton protein of the inner membrane is the mitochondrial calcium uniporter. Nature. 476, 336-340.doi:

$10.1038 /$ nature 10230

14. Duch, C., Mentel, T. and Pfluger, H. J. (1999).Distribution and activation of different types of octopaminergic DUM neurons in the locust. J. Comp. Neurol. 403, 119-134. DOI:10.1002/(SICI)10969861(19990105)403:1<119::AID-CNE9>3.0.CO;2-F

15. Eisner, V., Lenaers, G.andHajnóczky, G. (2014). Mitochondrial fusion is frequent in skeletal muscle and supports excitation-contraction coupling. J.Cell Biol. 205, 179195.doi: $10.1083 / j c b .201312066$.

16. El-Kholy, S., Stephano, F. , Li, Y. , Bhandari, A., Fink, C. and Roeder, T. (2015). Expression analysis of octopamine and tyramine receptors in Drosophila. Cell Tissue Res. 361(3), 669-684.doi: 10.1007/s00441-015-2137-4. Epub 2015 Mar 7.

17. Erion, R., DiAngelo, J. R., Crocker, A. and Sehgal, A. (2012). Interaction between sleep and metabolism in Drosophila with altered octopamine signaling. J. Biol.Chem. 287, 32406-32414.doi: 10.1074/jbc.M112.360875. Epub 2012 Jul 24.

18. Erspamer,V. andBoretti ,G. (1951). Identification and characterization, by paper chromatography, of enteramine, octopamine, tyramine, histamine and allied substances in extracts of posterior salivary glands of octopoda and in other tissue extracts of vertebrates and invertebrates, Arch IntPharmacodynTher. 88(3),296-332.

19. Evans, PD.(1981). Multiple receptor types of octopamine in the locust. J. physiol. 318,99-122.doi: 10.1113/jphysiol.1981.sp013853.

20. Fisher, J.A., Giniger, E., Maniatis, T and Ptashne, M. (1988). GAL4 activates transcription in Drosophila. Nature, 332(6167), 853-6.doi: 10.1038/332853a0.

21. Finkel, T. , 2011. Signal transduction by reactive oxygen species. J. Cell Biol. 194, 7-15.doi: $10.1083 /$ jcb.201102095.

22. Frezza, C., Cipolat, S., Martins de Brito, O., Micaroni, M., Beznoussenko, G.V., Rudka, T., et al (2006). OPA1 controls apoptotic cristae remodeling independently from mitochondrial fusion. Cell. 126, 177-189.doi: 10.1016/j.cell.2006.06.025.

23. Hoff, M., Balfanz, s., Ehling, P., Gensch, T. and Baumann, A. (2011). A single amino acid residue controls $\mathrm{Ca} 2+$ signaling by an octopamine receptor from Drosophila melanogaster. FASEB J. 25(7), 2484-2491. DOI:10.1096/fj.11-180703

24. Kelley, D. E., He, J., Menshikova, E. V. andRitov, V. B. (2002).Dysfunction of mitochondria in human skeletal muscle in type 2 diabetes.J.Diabetes. 51, 2944-2950.Doi:10.2337/diabetes.51.10.2944 
25. Khurana, S., Li, W-K.and Atkinson, N.S. (2010). Image enhancement for tracking the translucent larvae of Drosophila melanogaster.PloS ONE 5: e15259.doi:org/10.1371/journal.pone.0015259

26. Lee, H. G., Seong, C. S., Kim, Y. C., Davis, R. L. and Han, K. A. (2003). Octopamine receptor OAMB is required for ovulation in Drosophila melanogaster. Dev. Biol. 264(1), 179190.doi:org/10.1016/j.ydbio.2003.07.018

27. Li, Y., Hoffmann, J., Li, Y., Stephano, F., Bruchhaus, I., Fink, C. and Roeder, T. (2016). Octopamine controls starvation resistance, life span and metabolic traits in Drosophila. Sci. Rep. 6:35359. Doi:rg/10.1038/srep35359

28. Luo, J., Lushchak, O. V., Goergen, P., Williams, M. J. and Nässel, D. R. (2014). Drosophila insulinproducing cells are differentially modulated by serotonin and octopamine receptors and affect social behavior. PLoS One. 9:e99732.doi: 10.1371/journal.pone.0099732. eCollection 2014.

29. Martin, J.R., Ernst, R. , Heisenberg, M. (1998). Mushroom Bodies Suppress Locomotor Activity in Drosophila melanogaster. Learn. Mem.5(1), 179-191.

30. Maqueira, B., Chatwin, H. and Evans, P. D. (2005). Identification and characterization of a novel family of Drosophila beta-adrenergic-like octopamine G-protein coupled receptors. J. Neurochem. 94(2), 547-560.doi: 10.1111/j.1471-4159.2005.03251.x.

31. Orchard, I. and Lange, A.B. (1987). The release of octopamine and proctolin from an insect visceral muscle: effects of high-potassium saline and neural stimulation. Brain Res.J.413(2), 251-8.doi: 10.1016/0006-8993(87)91015-8.

32. Picard, M., Zhang, J., Hancock, S., Derbeneva, O., Golhar, R., Golik, P., et al (2014). Progressive increase in mtDNA 3243A > G heteroplasmy causes abrupt transcriptional reprogramming. PNAS.111, E4033-E4042.Doi:10.1073/pnas.1414028111

33. Porter, G.A., Makuck, R.F.andRivkees, S.A. (2002). Reduction in Intracellular Calcium Levels Inhibits Myoblast Differentiation. J. Biol. Chem. 277(32):28942-7.doi:org/10.1074/jbc.M203961200

34. Riemensperger, T., Voller, T., Stock, P., Buchner, E., Fiala, A., 2005. Punishment prediction by dopaminergic neurons in Drosophila.Curr. Biol. 15, 1953-1960.doi:10.1016/j.cub.2005.09.042.

35. Roeder, T. (1999). Octopamine in invertebrates.Prog.Neurobiol. 59(5),533-561.doi: 10.1016/s03010082(99)00016-7.

36. Roeder, T. (2002). Biochemistry and molecular biology of receptors for biogenic amines in locusts.Microsc. Res. Tech. 56(3),237-247.doi: 10.1002/jemt.10027.

37. Roeder, T. (2005). Tyramine and octopamine: ruling behavior and metabolism. Annu. Rev. Entomol. 50, 447-477.doi: 10.1146/annurev.ento.50.071803.130404.

38. Rothenfluh, A. and Heberlein, U. (2002). Drugs, flies, and videotape: the effects of ethanol and cocaine on Drosophila locomotion. Curr.Opin.Neurobiol. 12, 639-645. Doi:org/10.1016/S09594388(02)00380-X

39. Saini , A., Al-Shanti,N. andStewart, C. (2010). C2 Skeletal Myoblast Survival, Death, Proliferation and Differentiation: Regulation by Adra1d. Cell Physiol. Biochem. 25,253-262.doi: 10.1159/000276559. Epub 2010 Jan 12. 
40. Schwaerzel, M., Monastirioti, M., Scholz, H., Friggi-Grelin, F., Birman, S., Heisenberg, M.,

41. Dopamine and octopamine differentiate between aversive and appetitive olfactory memories in Drosophila. J. Neurosci. 23, 10495-10502.doi:org/10.1523/JNEUROSCI.23-33-10495.2003.

42. Sena, L. A. andChandel, N. S. (2012).Physiological roles of mitochondrial reactive oxygen species. Mol. Cell. 48, 158-167.doi: 10.1016/j.molcel.2012.09.025.

43. Sombati, S. and Hoyle, G. (1984).Generation of Specific Behaviors in a Locust by Local Release into Neuropil of the Natural Neuromodulator Octopamine.J .Neurobiol. 15(6), 481-506. Doi:org/10.1002/neu.480150607

44. Strauss, R. and Heisenberg, M .(1993). A Higher Control Center of LocomotorBehavior in the Drosophila Brain.J.Neurosci.13(5), 1852-1861. Doi:10.1523/jneurosci.13-05-01852.1993

45. Sujkowski, A., Gretzinger, A., Soave, N., Todi, S.V. and Wessells, R. (2020). Alpha- and beta-adrenergic octopamine receptors in muscle and heart are required for Drosophila exercise adaptations. Plos Genet. 16(6), e1008778.Doi: 10.1371/journal.pgen.1008778

46. Unoki, S., Matsumoto, Y., Mizunami, M., 2005. Participation of octopaminergic reward system and dopaminergic punishment system in insect olfactory learning revealed bypharmacological study. Eur. J. Neurosci. 22, 1409-1416.doi:org/10.1111/j.1460-9568.2005.04318.x

47. Zhou, C., Rao, Y., Rao, Y., 2008. A subset of octopaminergic neurons are important for Drosophila aggression. Nat. Neurosci. 11, 1059-1067.

\section{Figures}

\section{Figure 1}

Climbing speed of adult Drosophila males deficient in four different receptors receptive for octopamine as compared to controls. The experiment was performed in triplicate. Mean $\pm \mathrm{SD} ; \mathrm{N}=10 ;{ }^{\star} P \otimes 0.05, \star \star P \otimes 0.01$, $\star \star \star P \otimes 0.001$. 

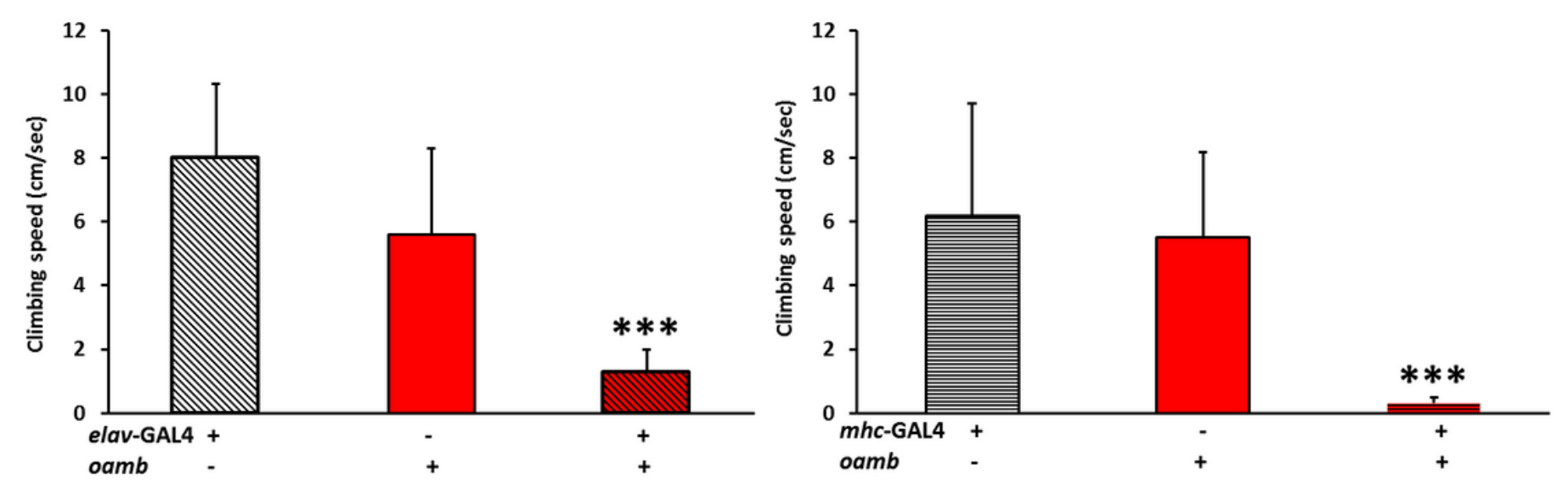

A

B

Figure 2

The climbing speed of oamb-deficient adult Drosophila males as compared to controls. The experiment was performed in triplicate.RNAi-mediated gene kockdown was directed to the nervous system using elav-GAL4 driver (A) and to the muscular system using mhc-GAL4 driver line (B). Three asterisks indicate high significance $(P \otimes 0.001)$.

Figure 3

Length and Width of fore and hind legs' parts of oamb-deficient as well as matching control adult Drosophila males.Mean $\pm \mathrm{SD} ; \mathrm{N}=10 ; * P \llbracket 0.05, * \star P \otimes 0.01, * \star \star P \otimes 0.001$.

Figure 4

Transmission electron micrograph of the leg muscular tissue in oamb-deficient adult Drosophila males . A and B TEM micrograph of male flies of matching controls of RNAi experiment. The muscles display features typical of insect skeletal muscle. $C$ and D, TEM micrograph of oambdeficient flies leg muscles. M, mitochondria.Myo, myofibrils.Z, z-line. 